\title{
CONDUCTAS ANTISOCIALES-DELICTIVAS Y SATISFACCIÓN FAMILIAR EN GRUPOS DE ESTUDIANTES DE QUINTO DE SECUNDARIA DE LIMA METROPOLITANA PERTENECIENTES A DIFERENTES ESTRATOS SOCIOECONÓMICOS
}

\author{
Carlos Ponce Díaz ${ }^{1}$
}

La presente investigación es de tipo correlacional y tuvo por finalidad estudiar si existía relación entre las conductas antisociales y la satisfacción familiar en los alumnos de quinto de secundaria de Lima Metropolitana, pertenecientes a diferentes estratos socio económicos. La muestra fue seleccionada a través de un muestreo no probabilístico de tipo intencionado y estuvo conformada por 1491 alumnos de ambos sexos, que cursaban el quinto año de secundaria en 20 Centros Educativos de Lima Metropolitana, de los cuales el $7.8 \%$ (116) fueron de clase alta; el 33.5\% (500), correspondieron a estudiantes de clase media; y el $58.7 \%$ (875), fueron escolares de nivel socio económico medio. Los instrumentos utilizados fueron: El Cuestionario A-D (Conductas Antisociales Delictivas), de Nicolás Seisdedos Cubero; y la Escala ESFA (Satisfacción Familiar por Objetivos), de 1. Barraca y L. López- Yarto, los cuales fueron adaptados en el presente reportando los análisis psicométricos realizados indicaron que ambos instrumentos presentaron confiabilidad, evaluada a través del método de consistencia interna. Asimismo, el análisis factorial exploratorio y los análisis de escalamiento multidimensional efectuados demostraron que las pruebas son válidas.

PALABRAS CLAVE: Conductas antisociales-delictivas; satisfacción familiar; estrato socio económico; estudiantes secundarios.

The present investigation is of corelational type and had by purpose of studying if existia relation between the conducts antisocial and the familiar satisfaction in the students of fifth of secondary one of Lima Metropolitan, pertaining to different economicos layers partner.

The sample was selected traves of a no probabilistico sampling of deliberate type and was conformed by 1491 students of both sexes, that attended fifth year de secondary in 20 Educative Centers of Lima Metropolitan, of which the 7,8\% (116) were of high class; the $33,5 \%$ (500), corresponded to students of middle-class; and the 58,7\% (875), were level students average economico partner. The used instruments were: The Questionnaire ACCEPTS (Antisociales-Delictivas Conducts), of Nicolas Seisdedos Cubero; and Scale ESFA (Satisfaction Familiar by Objectives), of J. Barraca and L. Lopez- Yarto, which was adapted in the present reporting the made psicometricos analisis indicated that both instruments presented/displayed trustworthiness, evaluated through metodo of internal consistency. Also, the exploratory factorial analisis and the conducted analisis of multidimensional escalamiento demonstrated that the tests are been worth.

KEY WORDS: Antisocial-criminal conducts; familiar satisfaction; layer economico partner; secondary students.

\footnotetext{
${ }^{1}$ Colaboradores: Luis Miguel Escurra Mayaute, Mario Bulnes Bedón; Jaime Aliaga Tovar; William Montgomery Urday; Eliana Delgado Coz y Leoncio Solórzano Acuña.
} 


\section{INTRODUCCIÓN}

Entre los adolescentes, las conductas antisociales y delictivas son un hecho que se detecta a nuestro alrededor y en diversas manifestaciones de la sociedad: en el cine; en la televisión; en la prensa; en la calle; en el centro educativo; en la universidad y, a veces desgraciadamente también en ambientes muy próximos a nosotros mismos y a nuestra familia.

Actualmente, el problema ya no es sólo de unos grupos marginados; se está extendiendo y aparece tanto en los centros urbanos como en los pequeños núcleos de población. Siempre han existido en algún grado esas conductas, pero actualmente se han multiplicado las oportunidades por circunstancias externas al mismo sujeto.

En determinados aspectos parece que el problema sólo obedece a factores ambientales, pero lo más probable, como ha sido señalado por diversos autores, es que, subyacente a todo ello, esté un determinado tipo de personalidad que facilite la desinhibición de los impulsos agresivos que, en principio, existen en todos los individuos.

En cuanto al desarrollo y maduración de este tipo de conducta, la edad de comienzo parece centrarse estadísticamente hacia los 15 años (Seisdedos, 1998), aunque ciertos comportamientos antisociales se hacen notar en la misma infancia. En ésta, los hechos delictivos (tales como el robo) son más frecuentes que lo que indican las estadísticas judiciales; existe una «cifra negra» de actos delictivos no registrados oficialmente que ha sido destacada por los criminólogos y que representa el volumen más importante de los comportamientos antisociales.

Aunque la delincuencia sancionada oficialmente es más abundante en los sujetos de niveles socioeconómicos bajos, también existe en los pertenecientes a estratos socio económicos altos, pero éstos, tal vez por su mayor cultura(resultado de unas mejores facilidades socioeconómicas), su delincuencia suele ser más sofisticada y encubierta, a la vez que, por el status de sus familias, sus actuaciones raras veces llegan a un tribunal(lo cual podría en parte, explicar algunas correlaciones negativas halladas en algunos medios entre el nivel socio económico y la delincuencia.

Son varias las disciplinas que, como la psicología, la criminología o la sociología, han intentado encontrar la causa de la aparición y el desarrollo de la Conductas Antisociales y la delincuencia: Wilson y Howel (1995) señalan como factores de riesgo determinadas características individuales, la influencia familiar y escolar, la influencia del grupo de pares y los efectos de la comunidad y el vecindario. Como factores de protección indican los lazos afectivos con la familia, la escuela y los pares y las creencias saludables de estándares de conducta adaptados.

Farrington (1995), según las conclusiones del Estudio de Cambridge, señala los más importantes predictores de la delincuencia. Otras clasificaciones (Buider, Geis, y Bruce, 1988; Elliot, Huizinga y Ageton, 1985; Goldstein, 1990; Hirschi, 1969; Patterson, de Barysche y Ramsey, 1989); recogen los siguientes factores: pobreza, bajo CI, acceso a las armas, consumo de drogas, predisposición genética, factores neurológicos y biológicos, debilidad de los lazos con instituciones sociales.

Respecto a las principales características de la Conductas Antisociales hay que señalar que presenta diferencias significativas de la conducta social normal (Kazdin y BuelaCasal, 1994).

Por otra parte, es importante señalar que la satisfacción familiar que experimenta un sujeto es un producto del continuo juego de interacciones (verbales y/o físicas) que mantiene con los otros miembros de su familia. Cuando las interacciones son reforzantes el sujeto tenderá a estar satisfecho, cuando sean punitivas tenderá a estar insatisfecho. 
Como resumen o resultante final de todo un conjunto de interacciones se mostrará global y bastante estable, siendo preciso un nuevo conjunto de interacciones para que cambien desde un polo (satisfacción) a otro (insatisfacción). La valoración que el sujeto formula sobre ella y en la que refleja esa resultante deberá contemplar necesariamente tanto aspectos cognitivos como, fundamentalmente, afectivos.

A fin de recabar correctamente esa valoración sobre la satisfacción familiar, los métodos de recogida deben tener en cuenta (y por lo tanto preguntar por) los sentimientos despertados ante la evocación de su familia y evitar al sujeto la elaboración de un juicio resumen personal-tarea cognitiva siempre más compleja- sobre la cuestión.

La satisfacción familiar se estudia, en este caso, no tanto como un juicio global expresado por el sujeto una vez comparada su realidad familiar con su ideal, sino como la sumatoria de distintos sentimientos que se despiertan en él al estar con su familia.

El presente estudio de tipo correlacional pretende responder a las siguientes interrogantes: ¿Presentan conductas antisocial es los estudiantes de quinto de secundaria pertenecientes a diferentes niveles socio económicos?, ¿Qué características de satisfacción familiar presentan los escolares de quinto de secundaria de Lima Metropolitana pertenecientes a distintos estratos socio económicos?, ¿Qué relación existe entre la Conductas Antisociales y la satisfacción familiar en grupos de estudiantes del quinto de secundaria y pertenecientes a diferentes niveles socio económicos?

\section{Objetivos}

a) Examinar la confiabilidad y validez del Cuestionario A-D de N. Seisdedos; y de la Escala ESFA, de J. Barraca, y L.Yarto.

b) Conocer las conductas antisociales y la satisfacción familiar que presentan los estudiantes del quinto de secundaria de Lima Metropolitana pertenecientes a distintos niveles socio económicos.

c) Detectar la existencia de diferencias significativas en las conductas antisociales, y en la satisfacción familiar, según el nivel socio económico al que pertenecen.

d) Investigar la existencia de diferencias significativas en las conductas antisociales, y en la satisfacción familiar, en virtud a las variables: sexo; edad cronológica; rendimiento escolar; y nivel socio económico.

\section{HIPÓTESIS}

$\mathrm{H}_{1}$ : Existe correlación negativa entre las conductas antisociales y la satisfacción familiar en los adolescentes del quinto de secundaria, en razón al estrato socio económico

$\mathrm{H} 2$ : Los alumnos del quinto de secundaria presentan conductas antisociales diferentes, según el estrato socio económico al que pertenecen

H3: Existen diferencias en la satisfacción familiar de los adolescentes del quinto de secundaria, en razón al nivel socio económico de procedencia.

\section{MÉTODO}

El diseño de investigación, corresponde al correlacional, pues se orienta a la determinación del grado de relación existente entre dos o más variables de interés en una misma muestra de sujetos o el grado de relación existente entre dos fenómenos o eventos observados.

Cuando se trata de una muestra de sujetos, el investigador observa la presencia o ausencia 
de las variables que desea relacionar y luego las relaciona por medio de la técnica estadística de análisis de correlación. En psicología por ejemplo, el investigador observará el momento en que aparece un determinado fenómeno, y qué otras circunstancias se presentan también de manera contemporánea para así poder determinar la posible relación existente entre dichos eventos.

Este tipo de estudio nos permite afirmar en qué medida las variaciones en una variable o evento están asociadas con las variaciones en la otra u otras variables o eventos. Asimismo, es importante señalar que este diseño permite un análisis estadístico de los resultados a través de un coeficiente de correlación, el cual adquiere valores que van desde -1 hasta + , teniendo el valor cero como intermedio. Cuando más se acerca el coeficiente a -1, la relación entre los eventos o variables observadas es inversa, es decir cuando se da una variable en una dirección, la otra tiene presencia en sentido contrario, y cuando más se acerca $a+1$, la relación es directa, es decir que la presencia en una dirección de una variable o evento cada vez más conlleva la presencia de la otra variable o evento en la misma dirección. El coeficiente cero indica ausencia total de relación. El esquema de este tipo de estudio es el siguiente:

$\begin{array}{ll} & \mathrm{Ox} \\ \mathrm{r} & \mathrm{Oy} \\ & \mathrm{r} \\ & \mathrm{Oz}\end{array}$

En este esquema $M$ es la muestra en la que se realiza el estudio y los subíndices x, y, z en cada $\mathrm{O}$ nos indican las observaciones obtenidas en cada una de tres variables distintas para el caso diagramado).

En nuestra investigación podemos apreciar que la hipótesis 1, formula la existencia de una posible correlación negativa entre las conductas antisociales y la satisfacción familiar en razón al estrato socioeconómico de procedencia de grupos de escolares de quinto de secundaria.

En un segundo momento se utilizará un diseño descriptivo comparativo, pues se trata de describir, y luego comparar por separado, las conductas antisociales y la satisfacción familiar, según el nivel socio económico; sexo; edad cronológica; y rendimiento escolar, al que pertenecen los alumnos examinados. Este segundo diseño parte de la consideración de dos o más investigaciones descriptivas simples; esto es, recolectar información relevante en varias muestras con respecto a un mismo fenómeno o aspecto de interés y luego caracterizar este fenómeno en base a la comparación de los datos recogidos, pudiendo hacerse esta comparación en los datos generales o en una categoría de ellos.

El esquema de este tipo de investigación corresponde al siguiente diagrama:

$\begin{array}{ll}\text { M1 } & \text { O1 } \\ \text { M2 } & \text { O2 } \\ & \text { O1=O2=O3=On } \\ \text { M3 } & \text { O3 } \\ \text { Mn } & \text { On }\end{array}$

Donde $M I, M 2, M 3, M n$, representan a cada una de las muestras; $O 1, O 2, O 3, O n$, la información (observaciones) recolectada en cada una de dichas muestras. Los $O 1$ a $O n$ en la parte lateral del diagrama nos indica las comparaciones que se llevan a cabo entre cada 
una de las muestras, pudiendo estas observaciones, resultados, o información ser: iguales, diferentes, o semejantes, con respecto a la otra. En nuestro estudio, podemos apreciar que las hipótesis 2 y 3 formulan la existencia de posibles diferencias significativas en las conductas antisociales, y en la satisfacción familiar, en grupos de escolares de tres estratos socioeconómicos diferenciados.

\section{Población y muestra de investigación}

La población estuvo conformado por los alumnos de quinto de secundaria matriculados en el año 2002 en los colegios estatales y particulares de Lima Metropolitana. Para el presente estudio se ha trabajado con una muestra representativa de 1491 casos, la cual fue seleccionada a través de un muestreo no-probabilístico de tipo intencional o intencionado.

En este tipo de muestreo quien selecciona la muestra lo que busca es que ésta sea representativa de la población de donde es extraída. Lo importante es que dicha representatividad se da en base a una opinión o intención particular de quien selecciona la muestra y por lo tanto la evaluación de la representatividad es subjetiva.

Para el presente estudio se han tomado muestras de 20 Centros Educativos de Lima Metropolitana, de los cuales, 6 de ellos, es decir el 33.5\% de la muestra corresponde a escolares de nivel socio económico bajo; 12 Colegios, es decir el 58.7\% de la muestra corresponde a sujetos de estrato socio económico medio; y dos Colegios, es decir, eI7.8\% de la muestra corresponde a jóvenes de nivel socio económico alto. Sobre el particular, debo indicar las dificultades que ha tenido mi equipo de investigadores para acceder a tomar muestras de Centros Educativos de niveles socio económicos altos.

\section{Composición de la Muestra}

En la tabla 1 podemos apreciar que un $47.4 \%$ de la muestra de estudiantes examinados son de sexo masculino y el $52.6 \%$ son de sexo femenino, lo cual nos habla de una muestra más o menos homogénea en lo que respecta al sexo.

\section{Tabla 1. Composición de la muestra por sexo}

\begin{tabular}{|l|l|c|}
\hline Sexo & Frecuencia & $\%$ \\
\hline Mujer & 707 & 47.40 \\
Varon & 784 & 52.60 \\
\hline Total & 1491 & 100.00 \\
\hline
\end{tabular}

En la tabla 2 podemos apreciar que la muestra ha oscilado entre los 14 y los 22 años de edad cronológica. Del total, el 52\% corresponde a estudiantes de 17 años; el $33.1 \%$ y $9.1 \%$ a alumnos con 16 y 18 años, respectivamente. En las edades antes mencionadas se encontraría el grueso de la muestra.

En la tabla 3 podemos apreciar que el $68 \%$ de la muestra ha tenido un rendimiento académico promedio durante la secundaria; el $21.5 \%$ un rendimiento entre los primeros; y finalmente, un $8.1 \%$ un rendimiento entre los últimos.

En la tabla 4 podemos apreciar que el $58.7 \%$ de la muestra examinada corresponden a escolares de nivel socio económico medio; el 33.5\% al nivel socio económico bajo; y el $7.8 \%$ al nivel socio económico alto. 
Tabla 2. Composición de la muestra por edad cronológica

\begin{tabular}{|l|l|l|}
\hline Edad & Frecuencia & $\%$ \\
\hline 14 & 1 & 0.10 \\
15 & 50 & $3.40(*)$ \\
16 & 493 & 33.10 \\
17 & 772 & 51.80 \\
18 & 136 & 9.10 \\
19 & 28 & 1.90 \\
20 & 4 & 0.30 \\
21 & 1 & 0.10 \\
22 & 1 & 0.10 \\
No responde & 5 & 0.30 \\
Total & 1491 & 100 \\
\hline
\end{tabular}

Tabla 3. Composición de la muestra por Rendimiento Académico

\begin{tabular}{|l|l|l|}
\hline Sexo & Frecuencia & $\%$ \\
\hline Rendimiento alto & 321 & 21.50 \\
Rendimiento promedio & 1014 & 68.00 \\
Rendimiento bajo & 121 & 8.10 \\
Sin datos & 35 & 2.30 \\
Total & 1491 & 10.00 \\
\hline
\end{tabular}

Tabla 4. Composición de la muestra por nivel socio económico

\begin{tabular}{|l|l|l|}
\hline Sexo & Frecuencia & $\%$ \\
\hline Bajo & 500 & 33.50 \\
Medio & 875 & 58.70 \\
Alto & 116 & 7.80 \\
Total & 1491 & 100.00 \\
\hline
\end{tabular}

\section{Variables del Estudio}

a. Estudio correlacional

- Variables correlacionadas

- Conductas antisociales.

- Satisfacción familiar.

b.Estudio descriptivo comparativo Variables independientes asignadas: - Estrato socio económico.

- Sexo.

- Edad cronológica.

- Rendimiento escolar.

Variables dependientes:

- Conductas antisociales.

- Satisfacción familiar. 


\section{Técnicas e Instrumentos de recolección de datos.}

a) Cuestionario A-D Conductas Antisociales-Delictivas, de Seisdedos Cubero, es producido por la Sección de Estudio de Tests de TEA Ediciones, S.A., Madrid(1987). En este caso hemos trabajado con la versión de 1998. El Cuestionario tiene una administración individual y colectiva; su tiempo de duración es variable oscilando entre los 10 y 15 minutos aproximadamente; puede ser aplicado en niños y adolescentes; Su Significación tiene que ver con la evaluación de dos aspectos, antisocial y delictivo, de la conducta desviada; y su Tipificación comprende Baremos para cada sexo en centiles y puntuaciones típicas. El cuestionario comprende un total de 40 ítemes (20 por cada uno de los aspectos que mide). En nuestro caso sólo hemos aplicado la parte correspondiente al aspecto de la Conductas Antisociales, adaptando algunas palabras que en la prueba original no hubiesen sido respondidas por los escolares peruanos tales como: «tacos» que significa «lisuras»; «gamberradas» que significa «palomilladas», respectivamente.

Una de las justificaciones que nos ha llevado a tomar sólo la parte referente a la medición de la Conductas Antisociales, tiene que ver con los índices de atracción, es decir, los elementos de la escala A (Antisocial) tienen mayor atracción que los de la escala D (Delictivo), por lo que, los sujetos admiten haber cometido más actos antisociales que delictivos. Si estos valores se consideran como índices de discriminación, como refiere el autor, la escala A es mucho más discriminativa (con valores cercanos al 50\% ), y sus distribuciones más cercanas a la de la curva normal, mientras que los índices de la escala D provocan unas distribuciones asimétricas de tipo positivo (pocos sujetos admiten haber cometido muchos actos delictivos). A esto se agrega que el ámbito de aplicación ha sido el educativo, en donde, los encargados del Colegio elegido para la aplicación, por lo general revisaron el material antes de ser administrado, de allí que, desde las primeras tomas, dicho personal se negó a damos facilidades frente a la aplicación de la Escala D.

b) Escala de Satisfacción Familiar por Adjetivos-ESFA, de Barraca y López- Yarto, es un instrumento publicado por TEA Ediciones, S.A. Madrid, 1997; tiene una aplicación individual y colectiva; su tiempo de duración es variable, aproximadamente 10 minutos; se puede aplicar a adolescentes y adultos; su significación se orienta a evaluar la satisfacción familiar expresada por los sujetos a través de distintos adjetivos.

Su tipificación se expone a través de Baremos españoles de población general (hombres y mujeres). En suma, dicha escala consta de 27 ítemes cada uno de los cuales está formado por una pareja de adjetivos antónimos. Esta estructura, propia de un diferencial semántico, confiere brevedad y sencillez a las contestaciones. La ESFA trata de evocar una respuesta preferentemente afectiva. Una única dimensión define la escala(satisfacción familiar), por lo que de su corrección se extrae una única puntuación.

La ESFA es una medida objetiva destinada a obtener una percepción global del sujeto respecto a su situación familiar. Puede utilizarse en la evaluación clínica CQmo instrumento complementario para conocer el bienestar en un área tan fundamental como es la familia, especialmente tras cambios normativos (por ejemplo: casamiento, hijos adolescentes, «nido vacío», jubilación, etc.) o nonormativos (por ejemplo: muerte repentina de algún miembro, cambio de residencia, desempleo, etc.). Aunque desde la escala se extrae una única puntuación, en el trabajo con caso único pueden examinarse más pormenorizadamente los adjetivos para detectar posibles causas concretas de la satisfacción o insatisfacción familiar, en virtud de los adjetivos señalados con puntuaciones extremas. En un primer análisis orientador, la escala ha demostrado su utilidad para distinguir entre una muestra control y una clínica en terapia familia, por lo que sería igualmente apreciable su uso pre y post tratamiento en una intervención cuyo objetivo terapéutico se centrase en 
mejorar las relaciones familiares. El otro gran campo de aplicación para la escala es el investigador. La puntuación en el instrumento puede servir como variable dependiente o variable independiente en distintas investigaciones que aprecien la importancia de una medida global en la satisfacción familiar.

Tanto el Cuestionario A-D (Conductas antisociales-delictivas) de Nicolás Seisdedos Cubero; y la Escala de Satisfacción Familiar por adjetivos-ESFA de J. Barraca y L. López Yarto, respectivamente. Fueron analizados por medio de los siguientes análisis psicométricos:
a) Análisis de la adecuación de los ítemes.
b)Aplicación piloto.
c) Administración masiva de las pruebas.
d)Estudio de la validez de constructo.
e)Estudio de la Confiabilidad.

Tabla 5 Análisis Psicométrico del Cuestionario de Conductas Antisociales.

\begin{tabular}{|c|c|c|c|}
\hline Item & $\mathbf{M}$ & D.E. & $r_{\text {it }}$ \\
\hline 1 & 0.42 & 0.49 & $0.56^{*}$ \\
\hline 2 & 0.45 & 0.50 & $0.50 *$ \\
\hline 3 & 0.37 & 0.48 & $0.51 *$ \\
\hline 4 & 0.21 & 0.11 & $0.48^{*}$ \\
\hline 5 & 0.71 & 0.46 & $0.49 *$ \\
\hline 6 & 0.46 & 0.50 & $0.51^{*}$ \\
\hline 7 & 0.55 & 0.50 & $0.40^{*}$ \\
\hline 8 & 0.57 & 0.48 & $0.50 *$ \\
\hline 9 & 0.31 & 0.46 & $0.43^{*}$ \\
\hline 10 & 0.36 & 0.48 & $0.47^{*}$ \\
\hline 11 & 0.26 & 0.44 & $0.30 *$ \\
\hline 12 & 0.27 & 0.44 & $0.51 *$ \\
\hline 13 & 0.41 & 0.49 & $0.54 *$ \\
\hline 14 & 0.37 & 0.48 & $0.44 *$ \\
\hline 15 & 0.29 & 0.45 & $0.43^{*}$ \\
\hline 16 & 0.53 & 0.50 & $0.45^{*}$ \\
\hline 17 & 0.64 & 0.48 & $0.42 *$ \\
\hline 18 & 0.36 & 0.48 & $0.51 *$ \\
\hline 19 & 0.45 & 0.50 & $0.47 *$ \\
\hline 20 & 0.53 & 0.50 & $0.49^{*}$ \\
\hline
\end{tabular}

f) Construcción de Normas o Baremos locales.

Complementariamente a los dos instrumentos mencionados, se ha aplicado un pequeño cuestionario de registro de variables independientes asignadas, en donde hemos utilizado lo referente al: estrato socio económico; el sexo; la edad cronológica; y el rendimiento escolar.

\section{Técnicas de procesamiento y análisis de datos}

El análisis estadístico ha comprendido los siguientes aspectos:

a) Análisis descriptivo y exploratorio de los datos, para describir de manera detallada la distribución de la muestra examinada; en tal sentido, se han calculado para las variables 
estudiadas: las frecuencias, los porcentajes, las medias o promedios aritméticos, las desviaciones Standard, así como los análisis de tallos y hojas y las pruebas de ajuste a la curva normal de Kolmogorov-Smirnov para los puntajes de las escalas de las pruebas.

b) Análisis psicométrico del Cuestionario de Conductas AntisocialesDelictivas A-D; y de la Escala de Satisfacción Familiar por Adjetivos ESFA, que incluye para ambos casos: el análisis de ítemes con el cómputo de las correlaciones ítem-test; el estudio de la validez deconstructo, que incluye el desarrollo del análisis factorial exploratorio, y un análisis factorial confirmatorio; y la con fiabilidad, cálculo de los coeficientes Alfa de Cronbach.

c) Análisis inferencial para la contrastación de hipótesis, para lo cual se ha ejecutado: la correlación de Pearson, el análisis de varianza de dos factores por nivel socio económico; sexo; edad cronológico; y rendimiento escolar, para cada uno de los aspectos investigados, y el análisis multivariado de varianza para cada grupo para determinar la jerarquía de las conductas antisociales y la satisfacción familiar.

\section{RESULTADOS}

A continuación se expondrán los principales resultados alcanzados:

\section{Análisis psicométrico del Cuestionario de Conductas Antisociales}

Los resultados presentados en la tabla 5 indican que los ítemes presentan correlaciones ítem-test corregidas que fluctúan entre 0.30 para el caso del ítem $11 ;$ y $0.56,0.54$, para los ítemes 1 y 13, lo cual corrobora que los ítemes son consistentes entre sí. Asimismo, el análisis de con fiabilidad por consistencia interna nos arroja un coeficiente alfa de 0.87 , que nos indica la existencia de puntajes con fiables.

Tabla 6. Análisis Factoríal Exploratorio del Cuestionario de Conductas Antisociales.

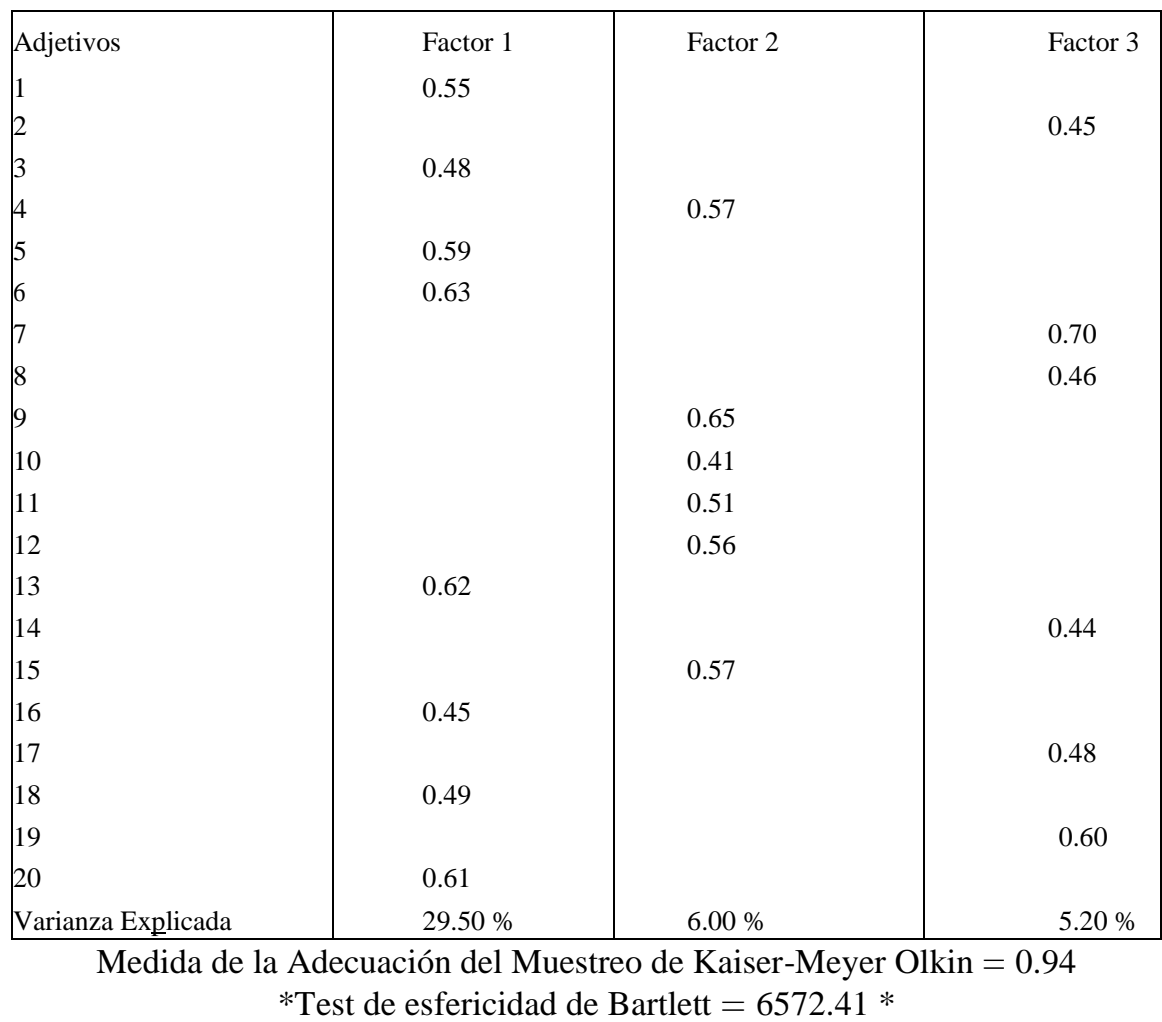

* Significativo $\mathrm{p}<0.05$

$\mathrm{N}=1491$ 
Los resultados de la exploración de la estructura factorial del cuestionario de conductas antisocial es revelan que la prueba estaría midiendo tres factores: un factor 1 compuesto por 8 itemes y que podríamos llamar ruptura de la interacción con otras personas; un factor 2 compuesto por 6 itemes y que podríamos llamar trasgresión de las normas convencionales; y un factor 3 compuesto por 6 ítemes y que podríamos llamar ruptura de la disciplina. 
Tabla 7. Análisis Psicométrico de los Factores del Cuestionario de Conductas Antisociales.

\begin{tabular}{|l|c|}
\hline Item & $\mathrm{r}_{\text {it }}$ \\
\hline Primer factor & \\
1 & $0.53^{*}$ \\
3 & $0.47^{*}$ \\
5 & $0.48^{*}$ \\
6 & $0.51^{*}$ \\
13 & $0.54^{*}$ \\
16 & $0.42^{*}$ \\
18 & $0.48^{*}$ \\
20 & $0.50^{*}$ \\
& Alfa de Crobach $=0.79^{*}$ \\
\hline Segundo factor & \\
4 & $0.44^{*}$ \\
9 & $0.46^{*}$ \\
10 & $0.39^{*}$ \\
11 & $0.29^{*}$ \\
12 & $0.46^{*}$ \\
15 & $0.41^{*}$ \\
& Alfa de Cronbach $=0.78^{*}$ \\
\hline Tercer factor & \\
7 & $0.43^{*}$ \\
8 & $0.41^{*}$ \\
14 & $0.43^{*}$ \\
17 & $0.39^{*}$ \\
\hline & $0.38^{*}$ \\
& $0.45^{*}$ \\
\hline
\end{tabular}

Los resultados obtenidos permiten apreciar que el cuestionario de conductas antisociales cuando es analizado por ítemes infiere la presencia de tres factores, los cuales tienen una confiabilidad significativa de: 079; 0.78; y 0.79, respectivamente, es decir, se trata de puntajes confiables. 
Análisis psicométrico de la escala de Satisfacción por Adjetivos

Tabla 8. Análisis Psicométrico de la Escala de satisfacción Familiar por Adjetivos.

\begin{tabular}{|c|c|c|c|}
\hline Item & $\mathrm{M}$ & D.E & $r_{i t}$ \\
\hline 1 & 4.71 & 0.92 & $0.63 *$ \\
\hline 2 & 4.33 & 1.22 & $0.63 *$ \\
\hline 3 & 4.35 & 1.11 & $0.52 *$ \\
\hline 4 & 1.45 & 1.07 & $0.63^{*}$ \\
\hline 5 & 4.01 & 1.38 & $0.63^{*}$ \\
\hline 6 & 3.99 & 1.33 & $0.32 *$ \\
\hline 7 & 4.42 & 1.16 & $0.72 *$ \\
\hline 8 & 4.51 & 1.34 & $0.66^{*}$ \\
\hline 9 & 4.69 & 1.13 & $0.66 *$ \\
\hline 10 & 4.56 & 1.10 & $0.65 *$ \\
\hline 11 & 4.20 & 1.38 & $0.56^{*}$ \\
\hline 12 & 4.36 & 1.18 & $0.70 *$ \\
\hline 13 & 4.22 & 1.23 & $0.56 *$ \\
\hline 14 & 4.48 & 1.28 & $0.74 *$ \\
\hline 15 & 4.09 & 1.24 & $0.72 *$ \\
\hline 16 & 4.12 & 1.41 & $0.64 *$ \\
\hline 17 & 4.34 & 1.23 & $0.55 *$ \\
\hline 18 & 4.57 & 1.27 & $0.70 *$ \\
\hline 19 & 4.28 & 1.27 & $0.61 *$ \\
\hline 20 & 4.54 & 1.20 & $0.55^{*}$ \\
\hline 21 & 4.19 & 1.35 & $0.65 *$ \\
\hline 22 & 4.63 & 1.12 & $0.67 *$ \\
\hline 23 & 4.07 & 1.19 & $0,50 *$ \\
\hline 24 & 4.41 & 1.24 & $0.65 *$ \\
\hline 25 & 4.26 & 1.34 & $0.71 *$ \\
\hline 26 & 4.41 & 1.18 & $0.62 *$ \\
\hline 27 & 4.69 & 1.34 & $0.74 *$ \\
\hline \multicolumn{4}{|c|}{ ALFA DE CRONBACH $=0.95^{*}$} \\
\hline
\end{tabular}

Los resultados obtenidos en la tabla 15, permiten apreciar que los ítemes presentan correlaciones item-test corregidas que fluctúan entre 0.32 y 0.56 para los ítemes 6 y 23 ; Y 0.74 para los ítemes 27 y 14, respectivamente, lo cual corrobora que los ítemes son consistentes entre si. Asimismo, el análisis de confiabilidad por consistencia interna nos arroja un coeficiente alfa de 0.95 , que nos indica la existencia de puntajes confiables 
Tabla 9. Análisis Factorial Exploratorio de la escala de Satisfacción Familiar por Adjetivos.

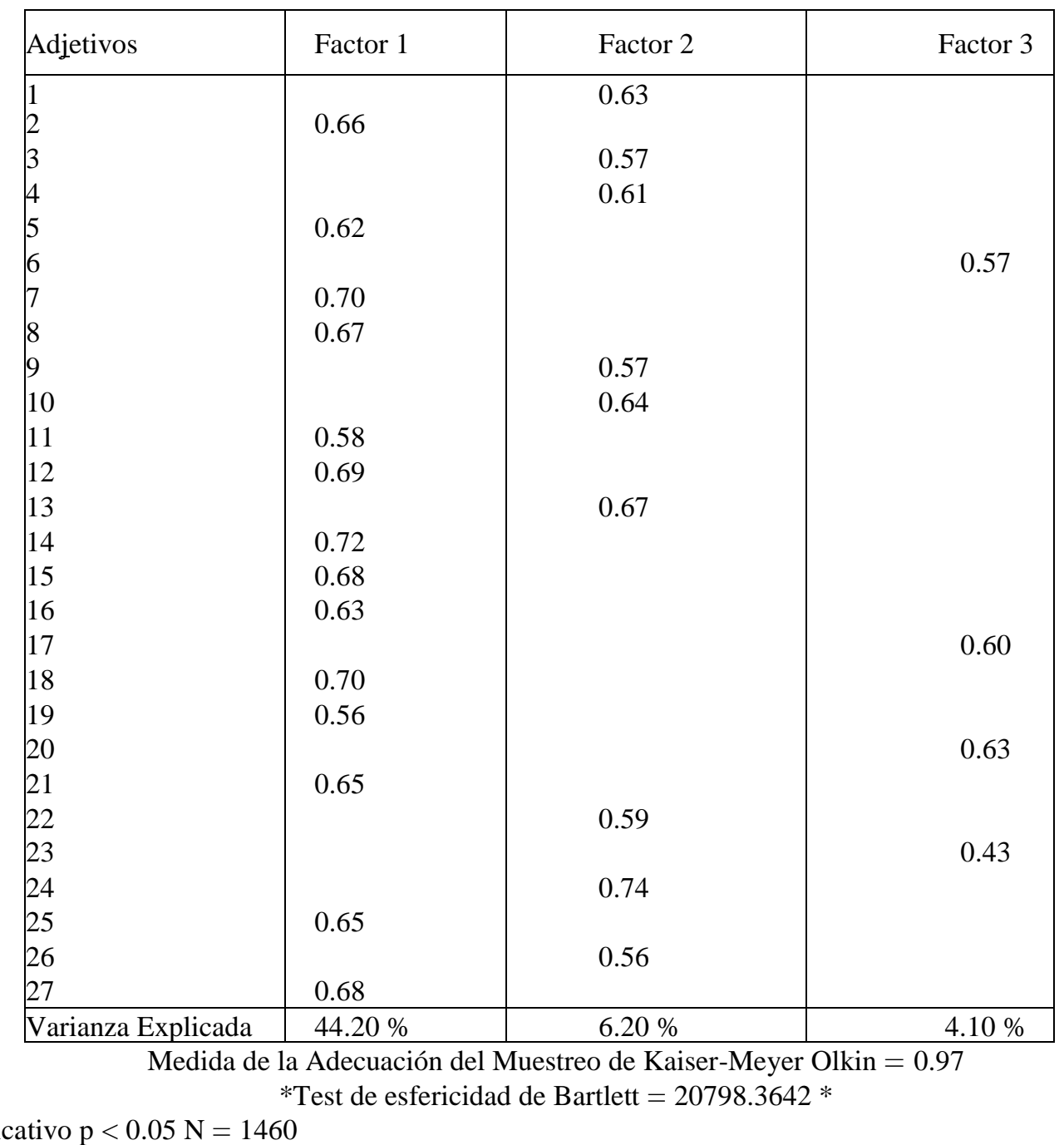

* Significativo $\mathrm{p}<0.05 \mathrm{~N}=1460$

Los resultados de la exploración de la estructura factorial de la escala de satisfacción familiar revelan que la prueba estaría midiendo tres factores: un factor 1 compuesto por 14 ítemes; un factor 2 compuesto por 9 ítemes; y un factor 3 comp'uesto por 4 ítemes, respectivamente. 
Tabla 10. Análisis Psicométrico de los Factores de la Escala de Satisfacción Familiar por Adjetivos.

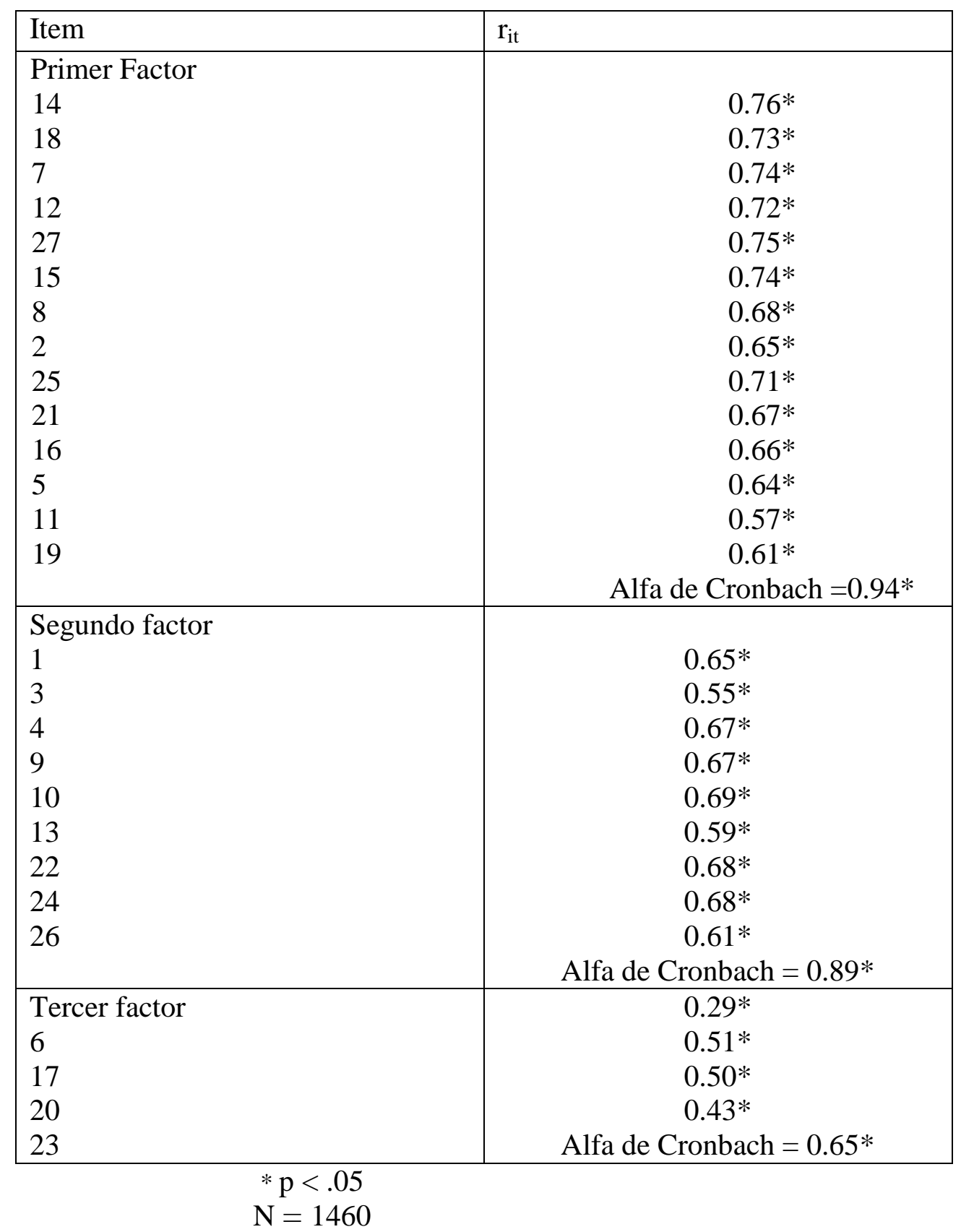

Los resultados obtenidos en el análisis factorial exploratorio, permiten apreciar que la escala de satisfacción familiar cuando es analizada por factores alcanzan valores de confiabilidad de 0.94 ; 0.89 ; y 0.65 , respectivamente, y por lo tanto se puede hablar de puntajes confiables en los tres factores. 


\section{Análisis descriptivo}

Tabla 11. Test de Bondad de Ajuste a la Curva Normal de KolmogorovSmirnov del Cuestionario de Conductas Antisociales.

\begin{tabular}{|l|llc|}
\hline Variable & Media & D.S. & K-S Z \\
\hline Factor 1 - CA & 3.73 & 2.47 & $4.11_{* * *}$ \\
Factor 2 -CA & 1.69 & 1.67 & $5.42 * * *$ \\
Factor 3 -CA & 3.02 & 1.84 & $4.87 * * *$ \\
Conductas Antisociales & 8.50 & 5.16 & $2.61 * * *$ \\
\hline
\end{tabular}

$* * * \mathrm{p}<0.001$

$\mathrm{N}=1491$

Los resultados obtenidos no se aproximan a la distribución normal, pues en todos los casos los valores del estadístico K-S Z, presentan niveles de significación inferiores a 0.05 , por 10 que es conveniente aplicar estadísticas no-paramétricas.

Tabla 12. Test de Bondad de Ajuste a la Curva Normal de KolmogorovSmirnov de la Escala de Satisfacción Familiar por Adjetivos.

\begin{tabular}{|l|lll|}
\hline Curso & Media & D.S. & K-S Z \\
\hline Factor 1 - SF & 60.59 & 13.40 & $3.10 * * *$ \\
Factor 2 - SF & 40.43 & 7.35 & $3.33 * * *$ \\
Factor 3 - SF & 16.95 & 3.45 & $3.41 * * *$ \\
Satisfacción Familiar Total & 117.97 & 21.90 & $2.34 * * *$ \\
\hline$* * \mathrm{P}<0.001$ & & & \\
$\mathrm{~N}=1460$ & & &
\end{tabular}

Los resultados observados indican que los puntajes no se aproximan a la distribución normal, pues en todos los casos los valores del estadístico K-S Z presenta probabilidades menores a 0.05), por lo que es recomendable analizar los datos con estadísticos no paramétricos. 


\section{Contraste de Hipótesis}

Tabla 13. Matriz de correlaciones entre las Satisfacción Familiar y las Conductas Antisociales

\begin{tabular}{|l|r|r|r|c|}
\hline Curso & Factor 1-CA & Factor 2-CA & Factor 3-CA & $\begin{array}{c}\text { Conductas } \\
\text { antisociales }\end{array}$ \\
\hline Factor 1 - SF & -0.01 & $-0.13 * * *$ & $-0.08 * *$ & $-0.08 * *$ \\
Factor 2 - SF & -0.07 & $-0.16 * * *$ & $-0.14 * * *$ & $-0.14 * * *$ \\
Factor 3 - SF & 0.02 & $-0.09 * * *$ & -0.03 & -0.03 \\
Satisfacción Familiar Total & -0.03 & $-0.15 * * *$ & $-0.10 * * *$ & $-0.10 * * *$ \\
\hline
\end{tabular}

$* * * \mathrm{P}<0.001$

$\mathrm{N}=1460$

En el análisis de la hipótesis 1 (ver Tabla 11), los resultados obtenidos permiten apreciar que se obtiene entre las conductas antisociales y la satisfacción familiar una correlación de - 0.10 , la cual es significativa, y presenta una fuerza baja, por lo que se puede concluir que a mayor satisfacción familiar existe menor tendencia a manifestar conductas antisociales en los escolares de quinto de secundaria examinados, y viceversa, por lo que se puede concluir que la Hipótesis es válida,

Tabla 14. Matriz de correlaciones entre las Satisfacción Familiar y las Conductas Antisociales del Grupo de Nivel Socio-económico Bajo.

\begin{tabular}{|l|c|c|c|c|}
\hline Curso & Factor 1-CA & Factor2-CA & Factor3-CA & $\begin{array}{c}\text { Conductas } \\
\text { antisociales }\end{array}$ \\
\hline Factor 1 - SF & -0.08 & $-0.12^{*}$ & $-0.11^{*}$ & $-0.12^{*}$ \\
Factor 2 - SF & $-0,09$ & $-0.17^{* * *}$ & $-0.18^{* * *}$ & $-0.16^{* * *}$ \\
Factor 3 - SF & -0.01 & $-0.09 * * *$ & -0.06 & -0.05 \\
Satisfacción Familiar & -0.08 & $-0.15^{* * *}$ & $-0.15^{* *}$ & $-0.14 * *$ \\
\hline
\end{tabular}

$* * * \mathrm{P}<0.001$

$\mathrm{N}=470$

Los resultados obtenidos revelan que en los escolares de nivel socio económico bajo, a menor satisfacción familiar tienden a manifestar una mayor tendencia de conductas antisociales.

Tabla 15. Matriz de correlaciones entre las Satisfacción Familiar y las Conductas Antisociales del Grupo de Nivel Socio-económico Medio.

\begin{tabular}{|l|c|r|r|c|}
\hline Curso & Factor 1-CA & Factor 2-CA & Factor 3-CA & $\begin{array}{c}\text { Conductas } \\
\text { antisociales }\end{array}$ \\
\hline Factor 1 - SF & $-0.14 * * *$ & $-0.19 * * *$ & $-0.17 * * *$ & $-0.19 * * *$ \\
Factor 2 - SF & $-0.17 * * *$ & $-0.18 * * *$ & $-0.18 * * *$ & $-0.20 * * *$ \\
Factor 3 - SF & -0.06 & $-0.10 * *$ & -0.06 & $-0.08 *$ \\
Satisfacción Familiar Total & $-0.15 * * *$ & $-0.19 * * *$ & $-0.17 * * *$ & $-0.19 * * *$ \\
\hline
\end{tabular}

$* * * \mathrm{P}<0.001$

$\mathrm{N}=874$ 
Los resultados obtenidos en los escolares de quinto de secundaria de nivel socioeconómico medio, revelan la misma tendencia que el resultado anterior de los de clase baja, es decir, a menor satisfacción familiar mayor incidencia de conductas antisociales, pero esta tendencia es más fuerte, ya que se está pasando de -0.14 a-0.19, respectivamente.

\section{Tabla 16. Matriz de correlaciones entre las Satisfacciones familiar y las conductas antisociales del grupo de nivel socioeconómica Alto.}

\begin{tabular}{|l|c|c|c|c|}
\hline Curso & Factor 1-CA & Factor2-CA & Factor3-CA & $\begin{array}{c}\text { Conductas } \\
\text { antisociales }\end{array}$ \\
\hline Factor 1 - SF & 0.02 & -0.10 & -0.15 & -0.09 \\
Factor 2 - SF & $-0,04$ & -0.12 & $-0.21^{*}$ & -0.15 \\
Factor 3 - SF & -0.05 & $-0.22 *$ & -0.13 & -0.17 \\
Satisfacción Familiar Total & -0.01 & -0.14 & -0.18 & -0.13 \\
\hline
\end{tabular}

*** $\mathrm{P}<0.001$

$\mathrm{N}=116$

Los resultados obtenidos en los estudiantes de nivel socioeconómico alto revelan que no existe correlación significativa entre la satisfacción familiar y las conductas antisociales

Tabla 17. Comparación de las conductas antisociales por nivel socio económico a través del análisis de varianza por Rangos de Kruskal-Wallis.

\begin{tabular}{|c|c|c|c|c|}
\hline \multirow[t]{3}{*}{ Variable } & \multicolumn{3}{|c|}{ Nivel Socio-cconómico } & \multirow{3}{*}{$X^{2}$} \\
\hline & Bajo & Medio & Alto & \\
\hline & $N=500$ & $\mathrm{~N}=875$ & $\mathrm{~N}=116$ & \\
\hline & $\mathrm{MR}$ & MR & MR & \\
\hline Factor $1-\mathrm{CA}$ & 569.86 & 799.67 & 1100.34 & $178.17^{* * 8}$ \\
\hline Factor $2-\mathrm{CA}$ & 714.84 & 752.89 & 828.33 & $7,46 *$ \\
\hline Factor $3-\mathrm{CA}$ & 632.39 & 787.68 & 921.31 & $63.69 * * *$ \\
\hline $\begin{array}{l}\text { Conductas } \\
\text { Antisociales }\end{array}$ & 605.23 & 790.39 & 1017.92 & $109.36^{* * *}$ \\
\hline${ }^{*} p<.05^{* *}$ & 0 & & & \\
\hline
\end{tabular}

Los resultados obtenidos tanto en el nivel general como en los factores específicos permiten apreciar la existencia de diferencias significativas en las conductas antisociales en relación al nivel socioeconómico, es decir, los alumnos de estrato socioeconómico alto evidencian conductas antisociales significativas en relación a los de los otros niveles socioeconómicos. Este resultado permite validar la hipótesis 2 
Tabla N 18. Comparación de la Satisfacción Familiar por nivel socio económico a través del análisis de varianza por Rangos de Kruskal-Wallis.

\begin{tabular}{|l|l|l|l|l|}
\hline Variable & \multicolumn{2}{|c|}{ Sexo } & & \\
& Bajo & Medio & Medio & $\mathrm{X}^{2}$ \\
& $\mathrm{~N}=470$ & $\mathrm{~N}=874$ & $\mathrm{~N}=116$ & \\
& MR & MR & MR & \\
\hline Factor1-CA & 566.68 & 791.32 & 935.99 & $116.77 * * *$ \\
Factor2-CA & 624.12 & 775.65 & 821.35 & $45.43 * * *$ \\
Factor3-CA & 609.14 & 781.81 & 835.59 & $59.61 * * *$ \\
\hline Satisfacción familiar & 567.2 & 794.76 & 906.29 & $110.64 * * *$ \\
\hline
\end{tabular}

Los resultados arrojan la presencia de diferencias significativas en la satisfacción familiar con respecto al nivel socio económico, es decir, los alumnos de estrato socio económico alto, presentan mayor satisfacción familiar. Estos hallazgos permite validar la hipótesis 3.

\section{Análisis complementarios}

Tabla 19. Comparación de las Conductas Antisociales por sexo a través de la Prueba U de Mann- Whitney.

\begin{tabular}{|l|l|l|l|l|}
\hline Variable & \multicolumn{2}{|c|}{ Sexo } & & \\
\hline & Varón & Mujer & U & Z \\
& N=707 & N=784 & & \\
& MR & MR & & \\
\hline Factor1-CA & 818.68 & 684.46 & 225759.59 & $6.23 * * *$ \\
Factor2-CA & 729.37 & 704.18 & 244357.00 & $4.05 * * *$ \\
Factor3-CA & 792.49 & 704.08 & 244276.00 & $4.01 * * *$ \\
\hline Conductas antisociales & 812.49 & 686.04 & 230139.00 & $567 * * *$ \\
\hline
\end{tabular}

Los resultados permiten apreciar que entre los estudiantes varones y mujeres tanto a nivel general como en cada uno de examinados. En tal sentido, los alumnos los factores, existen diferencias significativas varones tienden a presentar mayor número en la presencia de conductas antisociales de conductas antisociales que las mujeres.

Tabla 20 Comparación de la Satisfacción Familiar por sexo a través de la Prueba U de Mann- Whitney.

\begin{tabular}{|c|c|c|c|c|}
\hline Variable & \multicolumn{2}{|c|}{ Sexo } & & \\
\hline & $\begin{array}{l}\text { Varón } \\
\text { N=690 } \\
\text { MR }\end{array}$ & $\begin{array}{l}\text { Mujer } \\
\mathrm{N}=770 \\
\text { MR }\end{array}$ & $\mathrm{U}$ & $\mathrm{Z}$ \\
\hline Factor1-SF & 745.00 & 7.17 .51 & 255644.00 & 1.24 \\
\hline Factor2-SF & 749.97 & 713.05 & 252214.50 & 1.67 \\
\hline Factor3-SF & 748.97 & 714.05 & 252212.50 & 1.66 \\
\hline Satisfacción familiar & 748.62 & 714.26 & 253147.50 & 1.55 \\
\hline
\end{tabular}

Los resultados obtenidos en los tres factores y en el total, nos revelan que no existen diferencias significativas en la satisfacción familiar entre varones y mujeres. 
Tabla 21 Comparación de las conductas antisocial es por Edad a través del análisis de varianza por Rangos de Kruskal- Wallis.

\begin{tabular}{|l|lllllc|}
\hline Variable & \multicolumn{7}{|c|}{ Edad } \\
\hline & 15 & 16 & 17 & 18 & 19 & $X^{\prime}$ \\
& $\mathrm{N}=50$ & $\mathrm{~N}=493$ & $\mathrm{~N}=772$ & $\mathrm{~N}=136$ & $\mathrm{~N}=28$ & \\
& $\mathrm{MRR}$ & $\mathrm{MR}$ & $\mathrm{MR}$ & $\mathrm{MR}$ & $\mathrm{MR}$ & \\
\hline Factor I - CA & 579.29 & 654.26 & 810.78 & 731.86 & 624.68 & $50.91 * * *$ \\
Factor 2 - CA & 730.33 & 712.20 & 754.77 & 773.67 & 675.96 & 4.75 \\
Factor 3 - CA & 631.13 & 676.97 & 789.52 & 752.26 & 619.30 & $27.34 * * *$ \\
Conductas Antisociales & 608.74 & 666.49 & 797.80 & 753.56 & 609.20 & $36.35 * * *$ \\
\hline
\end{tabular}

$* \mathrm{p}<.05 * * \mathrm{p}<.01 * * * \mathrm{p}<.001$

Los resultados obtenidos permiten apreciar que en el total, existen diferencias significativas en la presencia de conductas antisociales entre los estudiantes varones y mujeres examinados. En tal sentido, 17 años es una edad pico o crítica a exhibir mayor efervescencia de conductas antisocial es, aunque los 16 y 18 años son edades cronológicas también significativas.

Tabla 22. Comparación de las conductas antisociales por nivel socio económico a través del análisis de varianza por Rangos de Kruskal- Wallis.

\begin{tabular}{|l|llllll|}
\hline Variable & \multicolumn{7}{|c|}{ Edad } \\
\hline & 15 & 16 & 17 & 18 & 19 & $\mathrm{X}^{2}$ \\
& $\mathrm{~N}=50$ & $\mathrm{~N}=480$ & $\mathrm{~N}=762$ & $\mathrm{~N}=133$ & $\mathrm{~N}=26$ & \\
& $\mathrm{MRR}$ & $\mathrm{MR}$ & $\mathrm{MR}$ & $\mathrm{MR}$ & $\mathrm{MR}$ & \\
\hline Factor 1 - SF & 657.52 & 708.69 & 771.49 & 612.08 & 426.98 & $34.23 * * *$ \\
Factor 2 - SF & 723.10 & 420.63 & 746.88 & 653.16 & 591.35 & $8.70 * * *$ \\
Factor 3 - SF & 711.03 & 702.26 & 757.98 & 669.93 & 542.62 & $13.52 * *$ \\
Conductas antisociales & 672.04 & 711.94 & 767.74 & 612.75 & 445.27 & $30.33 * * *$ \\
\hline
\end{tabular}

$* * \mathrm{p}<.01 \quad * * * \mathrm{P}<.001$

Los resultados revelan diferencias significativas en la satisfacción familiar respecto a la edad cronológica, es decir, los alumnos de 17 años presentan mayor satisfacción familiar que los extremos.

Tabla 23. Comparación de las conductas antisociales por nivel de Rendimiento Escolar económico a través del análisis de varianza por Rangos de Kruskal-Wallis.

\begin{tabular}{|l|l|l|l|l|}
\hline variable & \multicolumn{2}{|c|}{ Nivel de rendimiento escolar } & & \\
\hline & Bajo & Medio & Alto & $\mathrm{X}$ \\
& N=321 & N=1014 & N=121 & \\
& MR & MR & MR & \\
\hline Factor1-CA & 731.35 & 713.80 & 844.10 & $10.54^{* * *}$ \\
Factor2-CA & 709.56 & 718.05 & 866.28 & $15.04^{* * *}$ \\
Factor3-CA & 685.90 & 725.46 & 866.70 & $16.81^{* * *}$ \\
\hline $\begin{array}{l}\text { Conductas } \\
\text { antisociales }\end{array}$ & 709.33 & 717.22 & 873.90 & $15.92^{* * *}$ \\
\hline
\end{tabular}


Los resultados obtenidos en esta referencia estadística permiten apreciar que tanto en el total como en los factores específicos, los alumnos de menor rendimiento escolar tienden a manifestar conductas antisociales significativas en relación a los de rendimiento promedio y alto, respectivamente.

Tabla 24. Comparación de la Satisfacción Familiar por nivel de Rendimiento escolar a través del análisis de varianza por Rangos de Kruskal- Wallis.

\begin{tabular}{|l|l|l|l|l|}
\hline Variable & \multicolumn{4}{|c|}{ Nivel de rendimiento escolar } \\
& Bajo & Medio & Alto & $X^{2}$ \\
& N=320 & N=993 & N=114 & \\
& MR & MR & MR & \\
\hline Factor1-CA & 836.60 & 695.24 & 533.30 & $52.34 * * *$ \\
Factor2-CA & 836.67 & 694.85 & 536.47 & $51.77 * * *$ \\
Factor3-CA & 855.54 & 680.88 & 606.62 & $52.09 * *$ \\
\hline Satisfacción familiar & 851.54 & 690.90 & 529.09 & $1.74 * * *$ \\
\hline
\end{tabular}

Los resultados obtenidos revelan diferencias significativas en la satisfacción familiar con respecto al rendimiento escolar, es decir, los de mayor rendimiento exhiben mayor satisfacción familiar.

Tabla 25. Baremos del Cuestionario de Conductas Antisociales.

\begin{tabular}{|l|l|l|}
\hline Puntaje directo & Percentiles & Eneatipo \\
\hline 17 a mas & $96-100$ & 9 \\
$15-16$ & $89-95$ & 8 \\
$12-14$ & $77-88$ & 7 \\
$10-11$ & $60-76$ & 6 \\
$7-9$ & $40-59$ & 5 \\
$5-6$ & $23-39$ & 4 \\
$2-4$ & $11-22$ & 3 \\
1 & $4-10$ & 2 \\
0 & $0-3$ & 1 \\
\hline \multicolumn{2}{|c}{ M $=8.50$} \\
\hline
\end{tabular}

$\mathrm{N}=1491$

Tabla 26. Baremos de la escala de Satisfacción familiar

\begin{tabular}{|l|l|l|}
\hline Puntaje directo & Percentiles & Eneatipo \\
\hline 156 a mas & $96-100$ & 9 \\
$145-155$ & $89-95$ & 8 \\
$134-144$ & $77-88$ & 7 \\
$123-133$ & $60-76$ & 6 \\
$112-122$ & $40-59$ & 5 \\
$102-111$ & $23-39$ & 4 \\
$91-101$ & $11-22$ & 3 \\
$80-90$ & $4-10$ & 2 \\
$0-79$ & $0-3$ & 1 \\
\hline \multicolumn{3}{|c|}{ M $=8.50$} \\
& D.E $=5.16$ \\
\hline
\end{tabular}




\section{CONCLUSIONES}

1. Las conductas antisociales son más significativas en el estrato socio económico alto que en los otros niveles socio económicos,' lo cual estaría ratificando la hipótesis alternativa (1). 2.Los estudiantes de estrato socio económico alto, presentan mayor satisfacción familiar que los de los otros niveles socioeconómicos, lo cual estaría ratificando la hipótesis alternativa (2).

3. Los estudiantes de diferentes estratos socio económicos presentan distintos niveles de correlación entre la satisfacción familiar y las conductas antisociales, lo cual estaría corroborando la hipótesis (3).

4. En los grupos de escolares de quinto de secundaria: a mayor satisfacción familiar menor tendencia a manifestar conductas antisociales. En el análisis vemos que la correlación es baja, pero significativa.

5. Los varones y las mujeres presentan distintos valores de correlación entre la satisfacción familiar y las conductas antisociales.

6. En los escolares de nivel socio económico bajo, a menor satisfacción familiar tienden a manifestar una mayor tendencia a la Conductas Antisociales.

7. En los escolares de nivel socio económico medio, a menor satisfacción familiar mayor incidencia de Conductas Antisociales. Esta es más fuerte que la que presentan los de clase socioeconómica baja.

8. En los estudiantes de nivel socio económica alto, no existe correlación significativa entre la satisfacción familiar y la Conductas Antisociales.

Asimismo, es importante mencionar los siguientes hallazgos complementarios:

a. A nivel general, los alumnos varones tienden a presentar una mayor tendencia de conductas antisociales que las mujeres.

b. A nivel general, y en virtud a la edad cronológica, 17 años es la edad «pico» o crítica con respecto a exhibir conductas antisociales, aunque los 16 y 18 años son edades cronológicas también significativas.

c. A nivel general, y en virtud al rendimiento académico, los alumnos de menor rendimiento escolar tienden a manifestar conductas antisociales significativas en relación a los de rendimiento promedio y alto, respectivamente.

d. A nivel general, no existen diferencias significativas en la satisfacción familiar entre varones y mujeres.

e. A nivel general, existen diferencias significativas en la satisfacción familiar respecto a la edad cronológica en la muestra examinada, es decir, los adolescentes de 17 años presentan mayor satisfacción familiar que los de edades extremas.

f. A nivel general, existen diferencias significativas en la satisfacción familiar con respecto al rendimiento escolar, es decir, los de mayor rendimiento exhiben mayor satisfacción familiar.

g. Las Normas o Baremos obtenidos por eneatipos y percentiles nos están asegurando un elemento referencial propio de nuestra realidad y deben ser tenidos en cuenta por quienes utilicen el Cuestionario de Conductas Antisociales-Delictivas A-D; y la Escala de Satisfacción Familiar por Adjetivos ESFA. 


\section{Recomendaciones}

En el afán de conocer esta problemática a nivel nacional, se debe promover, no sólo estudios de replicación de la presente investigación; sino la realización de otros estudios considerando muestras de otras regiones del Perú especialmente de la sierra y de la selva, donde se podrían tener en cuenta además otras variables tales como las zonas: (rural y urbana), entre otras.

También sería conveniente elaborar modelos de intervención psicológica que puedan afrontar la problemática de las conductas antisociales y la satisfacción familiar en los escolares de Lima Metropolitana.

Finalmente, consideramos pertinente propiciar la realización de estudios multidisciplinarios que puedan enriquecer los hallazgos (educadores, sociólogos, y otros).

\section{REFERENCIAS BIBLIOGRAFICAS}

Barraca Mairal, J. (1997). Permanencia de los jóvenes en el hogar paterno: estudio de su satisfacción familiar desde un modelo integrador. Tesis Doctoral. Madrid: Universidad Pontificia Comillas.

Beavers, W.R. y Hampson, R. B. (1995).

Familias exitosas. Evaluación, tratamiento, e intervención. Buenos Aires; Paidós.

Eysenck H.J. Y Eysenck S.B.B (1981). EPQ-J. Cuestionario de la personalidad. Manual. Madrid: TEA.

Eysenck, S.B.G. y Seisdedos, N. (1978) Estudio internacional de la personalidad. Revista de Psicología General y Aplicada., 33, 271-281.

Morales, P. (1989). Medición de las actitudes en psicología y educación. Construcción de escalas y problemas metodológicos. San Sebastián: Tarttalo.

Seisdedos, N. (1982). Las Conductas Antisociales y delictiva de los adolescentes. Rev. SURGAM, 336, 11-17.

Yalverde Molina, J. Ma. (1980). El proceso de inadaptación social en el adolescente. Madrid: Facultad de Psicología. Univ. Complutense. Tesis doctoral (inédita), 1980. 